\title{
Effects of bufalin on the mTOR/p70S6K pathway and apoptosis in esophageal squamous cell carcinoma in nude mice
}

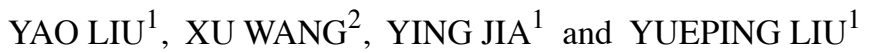 \\ ${ }^{1}$ Department of Pathology, The Fourth Hospital of Hebei Medical University, Shijiazhuang, Hebei 050011; \\ ${ }^{2}$ Department of Pathology, The First Central Hospital of Baoding, Baoding, Hebei 071000, P.R. China
}

Received June 29, 2016; Accepted June 15, 2017

DOI: $10.3892 /$ ijmm.2017.3039

\begin{abstract}
The aim of this study was to investigate the effects of bufalin on the mammalian target of rapamycin (mTOR/ p70S6 kinase (p70S6K) signaling pathway and cell apoptosis in orthotopically transplanted tumors in nude mice. The mice were inoculated with human esophageal squamous cell carcinoma (ESCC) ECA109 cells in order to establish a model of orthotopicall transplanted ESCC tumors. The mice are administered low, medium and high doses of bufalin $(0.5$, 1.0 and $1.5 \mathrm{mg} / \mathrm{kg}$ ) or rapamycin, or a combination of both. After the tumors were removed, the mRNA expression levels of mTOR, p70S6K, eukaryotic translation initiation factor 4E-binding protein 1 (4EBP1), cellular inhibitor of apoptosis protein 1 (cIAP1) and caspase-3 were detected by RT-PCR. In addition, we performed western blot analysis and immunohistochemical analysis to determine the protein expression of mTOR, p70S6K, 4EBP1, cIAP1, active caspase-3, Bcl-2 and Bad in the tumor tissue. The results revealed that bufalin exerted a significant anti-tumor effect in the nude mice with ESCC orthotopically transplanted tumors. This was shown by the decrease in the expression of mTOR, p70S6K and 4EBP1, which suggested that bufalin may possibly be used to inhibit tumor growth via the inhibition of the activation of p70S6K and 4EBP1. We also found that bufalin decreased the expression of cIAP1 and Bcl-2, and increased that of active caspase- 3 and Bad, thus indicating that bufalin promoted apoptosis. Thus, our findings suggest that bufalin promotes tumor cell apoptosis, and this may be one of the important anti-tumor mechanisms of action of bufalin.
\end{abstract}

\section{Introduction}

Esophageal carcinoma is the 6th frequent cause of cancerrelated mortality worldwide (1), and esophageal squamous cell

Correspondence to: Dr Yueping Liu, Department of Pathology, The Fourth Hospital of Hebei Medical University, Shijiazhuang, Hebei 050011, P.R. China

E-mail: annama@163.com

Key words: bufalin, mammalian target of rapamycin (mTOR/ p70S6 kinase (p70S6K) signaling pathway, apoptosis, esophageal squamous cell carcinoma, nude mice carcinoma (ESCC) accounts for $90 \%$ of all esophageal carcinoma cases in Asian countries (2). Although surgical techniques and peri-operative management have progressed, the prognosis for patients with ESCC remains poor (3). Thus, the identification of molecular therapeutic targets which may be used in the treatment of ESCC is one of the most promising avenues of research that lead to an improvement in the survival of patients with this type of refractory cancer. Some of the genetic alterations associated with the development or progression of ESCC have been described (4). However, few of these genes have been demonstrated to be associated with the biological or pathological characteristics of ESCC. Therefore, the identification of novel genes associated with the progression of ESCC is imperative.

The mammalian target of rapamycin (mTOR/p70S6 kinase (p70S6K) signaling pathway is an important intracellular signal transduction pathway, which is closely associated with cell survival and cell proliferation in many types of cancer. Studies have demonstrated that cell proliferation and cell cycle-related proteins (such as cyclin B1 and p27) $(4,5)$, cell apoptosis-related proteins [such as cellular inhibitor of apoptosis protein 1 (cIAP1) and caspase-3] $(6,7)$ and the key proteins [p70S6K and eukaryotic translation initiation factor 4E-binding protein 1 (4EBP1)] (8). Studies have shown that the mTOR pathway activates protein translation and regulates protein synthesis, which is the central regulator of cell growth. mTOR plays an important role in determining cell, tissue and organ size. The mTOR pathway is a key pathway that regulates cell growth and proliferation, which integrates signals from nutrient molecules, energy states and growth factors to regulate a large number of life processes, including autophagy $(9-10)$, ribose body biosynthesis $(11,12)$ and metabolism (13). p70S6K is the main downstream target of mTOR which make an important role in the translation initiation, protein synthesis, cell cycle, cell migration in the mTOR/ p70S6K pathway (19). The dysregulation of the pathway is associated with a variety of human diseases, including cancer $(14,15)$, diabetes (16) and leukemia (17,18). 4EBP1 is a downstream effector molecule of mTOR, through which (by phosphorylation) mTOR regulates cancer growth, proliferation and survival. cIAP1 is an inhibitor of apoptosis protein that inhibits apoptosis mainly by inhibiting the activity of caspase-3/7 and the role of nuclear factor $(\mathrm{NF})-\kappa \mathrm{B}(20)$. Caspase-3 is the most important terminal cleavage enzyme in the process of apoptosis. It is the terminal executive molecule downstream of the caspase cascade, which is the intersection of the death receptor pathway 
and mitochondrial pathway. Thus, caspase- 3 is also known as death protease (21). Furthermore, the mTOR/p70S6K pathway plays an important role in the growth, proliferation and antiapoptosis of ESCC (22).

The anticancer activity of cardiac glycosides (the main active ingredients in toad venom) has been a subject of investigation since 1967. Emerging evidence now suggests that cardiac glycosides are able to inhibit cell proliferation and induce the apoptosis of various tumor cell lines. Bufalin, a major bufadeinolide-like cardiac glycoside isolated from the skin and parotid venom glands of the toad (23), has been shown to induce cancer cell death through apoptotic pathways and has been investigted experimentally in a number of types of cancer.

The aim of this study was to evaluate the anti-tumor effects of bufalin by examining its effects on the mTOR/p70S6K signaling pathway and on cell apoptosis in a mouse model of ESCC created by the orthotopic transplanation of tumor cells. We examined the effects of bufalin on the expression of apoptosis-related proteins in order to determine the anti-tumor mechanisms of action of bufalin on a molecular level.

\section{Materials and methods}

Cells, antibodies and reagents. The ESCC cell line, ECA109, was provided by the Scientific Research Center of the Fourth Hospital of Hebei Medical University (Hebei, China). A total of $36 \mathrm{Nu} / \mathrm{Nu}$ nude mice (half male and half female, aged 4-6 weeks, weighed 18-22 g) purchased from Vital River Laboratory Animal Technology Co., Ltd. (Beijing, China). Our study was approved by the Ethics Committee of the Fourth Hospital of Hebei Medical University. Bufalin was obtained from Shanghai Yuanye Biotechnology Co., Ltd. (Shanghai, China) and rapamycin was purchased from the Beyotime Institute of Biotechnology (Shanghai, China). mTOR, p70S6K, 4EBP1, cIAP1, caspase-3, Bad, Bcl-2, $\beta$-actin upstream and downstream primers were all from Shanghai Sangon Biological Engineer ing Co., Ltd., (Shanghai, China). Rabbit anti-mTOR (1612-1), p70S6K (T2921), p-p70S6K (ab2571), 4EBP1 (1557-1), p-4EBP1 (2250-1), cIAP1 (3302-1), Bad (1541-1), Bcl-2 (1017-1) and $\beta$-actin monoclonal antibodies, and rat anti-active caspase- 3 monoclonal antibody (1476-1) were obtained from Epitomics (Burlingame, CA, USA). The Alpha Innotech UV gel imaging system was from Alpha Innotech Co., (San Leandro, CA, USA) and the Odyssey two-color infrared laser imaging system was from LI-COR Biosciences (Lincoln, NE, USA) The inverted phase contrast microscope was from Nikon (Tokyo, Japan).

Cell culture and tumor model. The ECA109 cells were seeded in $10 \%$ fetal bovine serum (FBS) of RPMI-1640 medium and incubated at $37^{\circ} \mathrm{C}$ in a $5 \% \mathrm{CO}_{2}$ incubator. The medium was changed once every 2 to 3 days. A total of 36 nude mice were inoculated with ECA109 cancer cells (each with $0.2 \mathrm{ml}$ injected subcutaneously into the right side of the axillary region) on the first day. The mice were provided with adequate food and water and kept in an environment with a temperature of $21 \pm 2^{\circ} \mathrm{C}$ and relative humidity of $30-70 \%$. No ECA109 cancer cells were injected during feeding. The mice were allowed to feed for 15 days and until the size of the tumors reached approximately $1 \mathrm{~cm}^{3}$. The mice were then randomly divided into 6 groups ( $n=6$ per group) as follows: the model group (saline- treated group), the low-dose bufalin group $(0.5 \mathrm{mg} / \mathrm{kg}$, BL group $)$, the medium-dose bufalin group ( $1 \mathrm{mg} / \mathrm{kg}$, BM group), the high-dose bufalin group (1.5 mg/kg, BH group), the rapamycin group (RAPA group) and the combination group (administered bufalin and rapamycin; BR group). The nude mice were injected for 30 days intraperitoneally according to the dose in each group every day apart from the combination group (once every other day). The mice in the model group were injected with $0.2 \mathrm{ml}$ saline once a day, and those in the rapamycin group were injected with $50 \mu \mathrm{g} / \mathrm{kg}$ rapamycin. The nude mice were sacrificed by cervical dislocation at the end of the treatment period. The tumors were then removed and approximately $0.5 \mathrm{~cm}^{3}$ of the tumor was cut and placed in a refrigerator at $-80^{\circ} \mathrm{C}$ for use in RT-PCR and western blot analysis. The remaining parts of the tumors were fixed in formalin for H\&E staining, TUNEL and immunohistochemistry. The tumor inhibition rate was calculated as follows: tumor inhibition $(\%)=$ (mean tumor volume of model group - mean tumor volume of intervention group)/mean tumor volume of model group x $100 \%$.

Morphologicalanalysis. Thegradedethanol-dehydrated,paraffinembedded, hematoxylin and eosin (H\&E)-stained, xylene-cleared tissue sections were sliced at $4 \mu \mathrm{m}$. The H\&E-stained sections were observed under a microscope (Axio Scope.A1; Carl Zeiss (Shanghai) Management Limited, Shanghai, China), and the groups were divided according to the necrosis range, that is, focal necrosis, a large area of necrosis, or a larger area of necrosis.

TUNEL assay. The tumor tissues were fixed in $10 \%$ neutral formalin, dehydrated, paraffin-embedded and sectioned prior to TUNEL staining. The sections were then digestived with $20 \mu \mathrm{g} / \mathrm{ml}$ proteinase $\mathrm{K}$ (Beyotime Institute of Biotechnology) for $15 \mathrm{~min}$. The apoptotic index in the tumor tissue was detected using the ApopTag Peroxidase In Situ Apoptosis Detection kit (EMD Millipore; Bilerica, MA, USA). The results of TUNEL staining were observed under a microscope (Axio Scope.A1; Carl Zeiss (Shanghai) Management Limited). The positive staining was observed in DAB coloring liquid. A total of 10 high power field (HPF) were selected to calculate the proportion of positive cells randomly and the apoptotic index (AI) was the number of positive cells in 100 cells.

$R T-P C R$. Total RNA was extracted using TRIzol reagent (Sangon Biotech (Shanghai) Co., Ltd., Shanghai, China) and the integrity of RNA was determined using the Alpha Innotech Gel imaging system. Total RNA were reverse transcribed into cDNA. At the end of PCR amplification, the PCR amplification products were confirmed and analyzed. The primers for mTOR, p70S6K, 4EBP1, cAIP1, caspase-3 primers were verified by NCBI and $\beta$-actin was used as an internal reference gene. The primer sequences were as follows: mTOR (318 bp) forward, 5'-AGTGGACCAGTGGAAAC AGG-3' and reverse, 5'-TTCAGCGATGTCTTGTGAGG-3'; p70S6K (188 bp) forward, 5'-TACTTCGGGTACTTGGTAA-3' and reverse, 5'-GATGAAGGGATGCTTTACT-3'; 4EBP1 (156 bp) forward, 5'-ACCGGAAATTCCTGATGGAG-3' and reverse, 5'-CCCGCTTATCTTCTGGGCTA-3'; cIAP (245 bp) forward, 5'-TCCCAGGTCCCTCGTATCAA-3' and reverse, 5'-ATCCAGCATCAGGCCACAAC-3'; caspase-3 (116 bp) forward, 5'-TCATTATTCAGGCCTGCCGTGGTA-3' and 
Table I. The mental state and dietary habits of the nude mice during the course of medication.

Dietary habits

\begin{tabular}{lccr} 
Groups & Mental state & Fodder consumption $(\mathrm{g})$ & Post-treatment \\
\hline Model & Good & $3.2 \pm 0.3$ & $4.5 \pm 0.1$ \\
Low-dose bufalin & Good & $3.4 \pm 0.3$ & $4.4 \pm 0.3$ \\
Medium-dose bufalin & Good & $3.5 \pm 0.2$ & $4.6 \pm 0.3$ \\
High-dose bufalin & Good & $3.8 \pm 0.2$ & $4.9 \pm 0.2$ \\
Rapamycin & Good & $3.4 \pm 0.3$ & $4.6 \pm 0.5$ \\
Combination & Good & $3.6 \pm 0.2$ & $4.6 \pm 0.1$
\end{tabular}

Values are expressed as the means $\pm \mathrm{SD}(\mathrm{n}=3)$. Pairwise comparision between each group, $\mathrm{P}>0.05$.

reverse, 5'-ATGCATACAAGAAGTCGGCCTCCA-3'. The reaction conditions were as follows: for $\mathrm{mTOR}: 95^{\circ} \mathrm{C}$ for $5 \mathrm{~min}$, $95^{\circ} \mathrm{C}$ for $30 \mathrm{sec}, 60.1^{\circ} \mathrm{C}$ for $30 \mathrm{sec}, 72^{\circ} \mathrm{C}$ for $30 \mathrm{sec} ; 37 \mathrm{cycles}$, $72^{\circ} \mathrm{C}$ for $10 \mathrm{~min}$; for $\mathrm{p} 70 \mathrm{~S} 6 \mathrm{~K}$ : $95^{\circ} \mathrm{C}$ for $5 \mathrm{~min}, 95^{\circ} \mathrm{C}$ for $45 \mathrm{sec}$, $48^{\circ} \mathrm{C}$ for $45 \mathrm{sec}, 72^{\circ} \mathrm{C}$ for $1 \mathrm{~min}, 37$ cycles; $72^{\circ} \mathrm{C}$ for $5 \mathrm{~min}$; for 4EBP1: $95^{\circ} \mathrm{C}$ for $5 \mathrm{~min}, 95^{\circ} \mathrm{C}$ for $30 \mathrm{sec}, 48^{\circ} \mathrm{C}$ for $30 \mathrm{sec}, 72^{\circ} \mathrm{C}$ for $30 \mathrm{sec}, 37$ cycles; $72^{\circ} \mathrm{C}$ for $10 \mathrm{~min}$; for cIAP1: $95^{\circ} \mathrm{C}$ for $5 \mathrm{~min}, 95^{\circ} \mathrm{C}$ for $30 \mathrm{sec}, 60^{\circ} \mathrm{C}$ for $50 \mathrm{sec}, 72^{\circ} \mathrm{C}$ for $30 \mathrm{~min}$, 35 cycles; $72^{\circ} \mathrm{C}$ for $5 \mathrm{~min}$; caspase- 3 was $95^{\circ} \mathrm{C}$ for $5 \mathrm{~min}, 95^{\circ} \mathrm{C}$ for $30 \mathrm{sec}, 56^{\circ} \mathrm{C}$ for $45 \mathrm{sec}, 72^{\circ} \mathrm{C}$ for $1 \mathrm{~min}, 35$ cycles; $72^{\circ} \mathrm{C}$ for $10 \mathrm{~min}$. The results were observed using the Alpha Innotech UV gel imaging system and the grayscale value was then calculated as follows: relative expression amount $=$ grayscale value of target gene/grayscale value of $\beta$-actin.

Western blot analysis. Approximately $30 \mathrm{mg}$ fresh tumor tissue was ground and place on ice. This was followed by the addition of $300 \mu \mathrm{l}$ ice-cold lysis buffer and $2 \mu \mathrm{l}$ PMSF. The samples were then centrifuged at $12,000 \times \mathrm{g}$ at $4^{\circ} \mathrm{C}$ for $15 \mathrm{~min}$. The proteins $(50 \mu \mathrm{g})$ were denatured at $95^{\circ} \mathrm{C}$ in metal bath and then subjected to SDS-PAGE and transferred onto PVDF membranes. The membranes were then incubated with the primary antibodies, mTOR, p70S6K, p-p70S6K, 4EBP1, p-4EBP1, cIAP1, active caspase-3, Bad and Bcl-2 (dilution, 1:2,000), diluted in TBST overnight at $4^{\circ} \mathrm{C}$ in a shaker. We then used an Odyssey two-color infrared laser imaging system to capture and analyze the images after washing the membranes 3 times with TTBS and the grayscale value was then determined.

Immunohistochemistry. Immunohistochemistry was carried out using the EnVision ${ }^{\mathrm{TM}}$ immunohistochemistry kit (Agilent Technologies, Santa Clara, CA, USA) according to the manufacturer's instructions. This was followed by the addition of antibodies (mTOR, p70S6K, p-p70S6K, 4EBP1, p-4EBP1, cIAP1, active caspase-3, Bcl-2 and Bad) to the slices and incubation at room temperature for $150 \mathrm{~min}$, paying attention to ensure that the antibodies were in full contact with the tissues. We determined the location of mTOR, p70S6K, p-p70S6K, 4EBP1, p-4EBP1, cIAP1, active caspase-3, Bcl-2 and Bad on the cell membrane, nucleus or cytoplasm. The staining intensity was scored as follows: no color corresponding to 0 , canary yellow corresponding to 1 , pale brown corresponding to 2 , tan corresponding to 3 ; the percentage of positive cells
Table II. Average weight of nude mice in the transplanted model before and after treatment.

\begin{tabular}{lcc}
\hline & \multicolumn{2}{c}{ Body weight $(\mathrm{g})$} \\
\cline { 2 - 3 } Groups & Pre-treatment & Post-treatment \\
\hline Model & $20.9 \pm 2.4$ & $27.9 \pm 3.0$ \\
Low-dose bufalin & $20.8 \pm 1.5$ & $28.2 \pm 1.9$ \\
Medium-dose bufalin & $20.8 \pm 2.1$ & $27.9 \pm 2.9$ \\
High-dose bufalin & $20.9 \pm 2.7$ & $28.1 \pm 2.8$ \\
Rapamycin & $20.9 \pm 1.9$ & $27.9 \pm 2.0$ \\
Combination & $20.8 \pm 2.1$ & $27.8 \pm 2.6$ \\
\hline
\end{tabular}

Values are expressed as the means $\pm \mathrm{SD}(\mathrm{n}=3)$. Pairwise comparision between each group, $\mathrm{P}>0.05$.

was negative corresponding to 0 , positive cells $<10 \%$ corresponding to 1 , positive cells between $11-50 \%$ corresponding to 2 , positive cells between $51-75 \%$ corresponding to 3 , and positive cells $>75 \%$ corresponding to 4 . The product of staining intensity and the percentage of positive cells was negative (-) corresponding to 0 , weakly positive (+) corresponding to $1-4$, moderate positive $(++)$ corresponding to 5-8 and strong positive $(+++)$ corresponding to $9-12$.

Statistical analysis. One-way analysis of variance (ANOVA) was used to determine the differences between the 6 groups and multiple comparisons between groups were made using the Student-Newman-Keuls (SNK) test. The experimental data of the results of immunohistochemistry were examined using the Kruskal-Wallis $\mathrm{H}$ test and multiple comparisons between groups were made using the Nemenyi test. The results were considered statistically significant at a vaue of $\mathrm{P}<0.05$.

\section{Results}

No obvious abnormalities were observed as regards the dietary habits of the nude mice diet and the mental state of the mice in all the groups (model, BL, BM, BH, RAPA and BR) during the course of medication $(\mathrm{P}>0.05)$ (Table I). There were no significant differences in the body weight of the mice in 
Table III. Average tumor volume and inhibiting rate of the transplanted mode before and after treatment.

Tumor volume $\left(\mathrm{cm}^{3}\right)$

Groups

Pre-treatment

Post-treatment

Tumor inhibition (\%)

\begin{tabular}{lllr}
\hline Model & $0.199 \pm 0.103$ & $1.778 \pm 0.176$ & - \\
Low-dose bufalin & $0.194 \pm 0.111$ & $1.714 \pm 0.188$ & 3.6 \\
Medium-dose bufalin & $0.206 \pm 0.099$ & $1.115 \pm 0.124^{\mathrm{a}}$ & 37.3 \\
High-dose bufalin & $0.197 \pm 0.079$ & $0.713 \pm 0.122^{\mathrm{a}}$ & 59.9 \\
Rapamycin & $0.192 \pm 0.126$ & $0.699 \pm 0.079^{\mathrm{a}}$ & 60.7 \\
Combination & $0.203 \pm 0.121$ & $0.506 \pm 0.076^{\mathrm{a}}$ & 71.5 \\
\hline
\end{tabular}

Values are expressed as the means $\pm \operatorname{SD}(n=3)$. ${ }^{\mathrm{a}} \mathrm{P}<0.05$, vs. model group.

each group prior to treatment $(\mathrm{P}>0.05)$, and even post-treatment $(\mathrm{P}>0.05)$ (Table II). The tumor size of the mice in each group (model, BL, BM, BH, RAPA and BR) was $1.778 \pm 0.176$, $1.714 \pm 0.188,1.115 \pm 0.124,0.713 \pm 0.122,0.699 \pm 0.079$ and $0.506 \pm 0.076 \mathrm{~cm}^{3}$, respectively and the tumor inhibition rate was 4.1, 37.6, 60.1, 60.9 and $71.7 \%$, respectively (Fig. 1, Table III). It proved that bufalin has obvious antitumor effect.

Morphology of cells in tumor tisue. We observed the morphological changes in the tumor tissue isolated from the nude in each group mice with orthotopically transplanted tumors. The hematoxylin and eosin (H\&E)-stained sections were observed under a microscope. Tumor necrosis was evident in all the groups, apart from the model group in which necrosis was hardly evident. The tissues from the BL group exhibited the most extensive focal necrosis; the tisues from the BM group exhibited a large area of necrosis; the tissues from the BH, RAPA and BR groups exhibited large areas of necrosis and cancer cell clumps were scattered in the necrotic tissues (Fig. 2).

Bufalin promotes cell apoptosis in ESCC tumor tissue, as shown by TUNEL assay. Apoptotic nuclei were stained brownish yellow. The apoptotic indexes of each treatment groups (BL, BM, BH, RAPA and BR) were significantly higher $(13.67,16.17,10.83,10.33$ and $8.5 \%)$ than those of the model group (2.17\%); those of the BM group were the highest (Fig. 3).

Bufalin affects the expression of apoptosis-related genes. The mRNA expression levels of mTOR and cIAP1 gradually decreased in the treatment groups (BL, BM, BH, RAPA and BR) compared to the model group, whereas the mRNA expression levels of caspase-3 gradually increased; significant differences were observed $(\mathrm{P}<0.05)$. It should be noted that no significant differences were observed between the model and BL groups $(\mathrm{P}>0.05)$, and between the BH and RAPA groups $(\mathrm{P}>0.05)$. The mRNA expression levels of $\mathrm{p} 70 \mathrm{~S} 6 \mathrm{~K}$ and 4EBP1 did not differ significantly between the groups $(\mathrm{P}>0.05$; model, BL, BM, BH, RAPA and BR) (Fig. 4).

Bufalin increases the expression of apoptotic proteins, and decreases that of anti-apoptotic proteins. The protein expression levels of mTOR, p-p70S6K, p-4EBP1, cIAP1

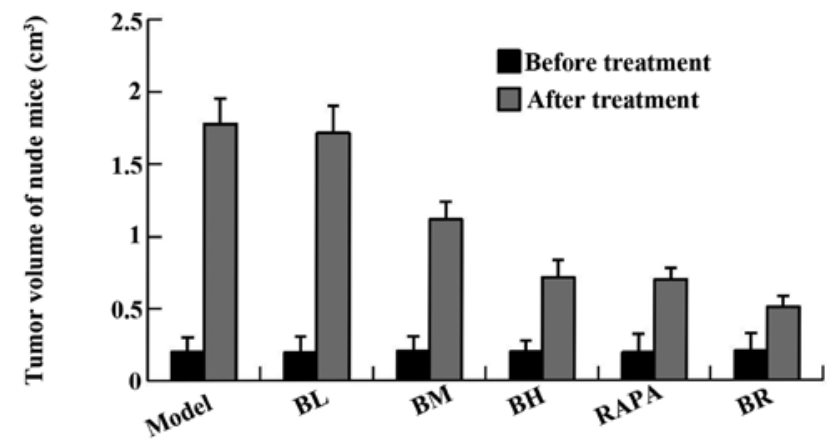

Figure 1. The change of nude mice tumor volume before and after treatment. ${ }^{*} \mathrm{P}<0.05$ vs. model.

and Bcl-2 gradually decreased in the treatment groups (BL, BM, $\mathrm{BH}, \mathrm{RAPA}$ and BR) compared to the model group. However, the protein levels of caspase-3 and Bad gradually increased, with significant differences between the each groups $(\mathrm{P}<0.05)$. Again, it should be noted that no significant differences were observed between the model and BL groups $(\mathrm{P}>0.05)$, and between the $\mathrm{BH}$ and RAPA groups $(\mathrm{P}>0.05)$. No statistically significant differences were observed in the protein expression levels of p70S6K and 4EBP1 (P>0.05) between the (model, BL, BM, BH, RAPA and BR) (Fig. 5).

Immunohistochemistry assay. The immunoreactive scores for mTOR, p-p70S6K, p-4EBP1, cIAP1 and Bcl-2 gradually decreased in each treatment group (BL, BM, BH, RAPA and BR) (Fig. 6), whereas those for active caspase-3 and Bad gradually increased compared to the model group (Fig. 7), with significant differences observed $(\mathrm{P}<0.05)$. However, no significant differences were observed between the model and $\mathrm{BL}$ groups $(\mathrm{P}>0.05)$, and between the BH and RAPA groups $(\mathrm{P}>0.05)$. The immunoreactive scores for $\mathrm{p} 70 \mathrm{~S} 6 \mathrm{~K}$, 4EBP1 did not differ significantly between the groups (model, BL, BM, BH, RAPA and BR (P>0.05) (Fig. 8).

\section{Discussion}

Bufalin is one of the main components extracted from toad venom and is used in traditional Chinese medicine (TCM) (23). It is known to exert anti-tumor effects. Bufalin has been shown 

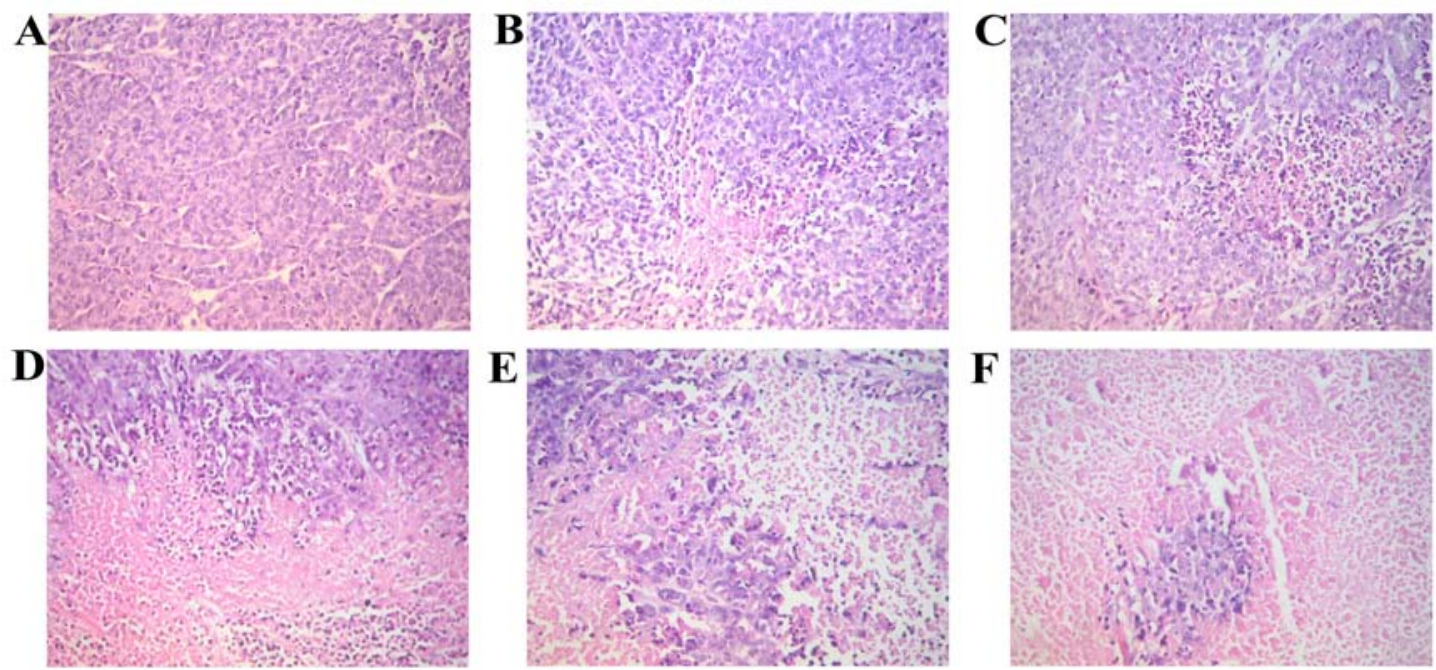

Figure 2. The morphology of nude mice transplanted tumor (by H\&E staining, x20 magnification). (A) Model; (B) low-dose bufalin; (C) medium-dose bufalin; (D) High-dose bufalin; (E) rapamycin and (F) combination.
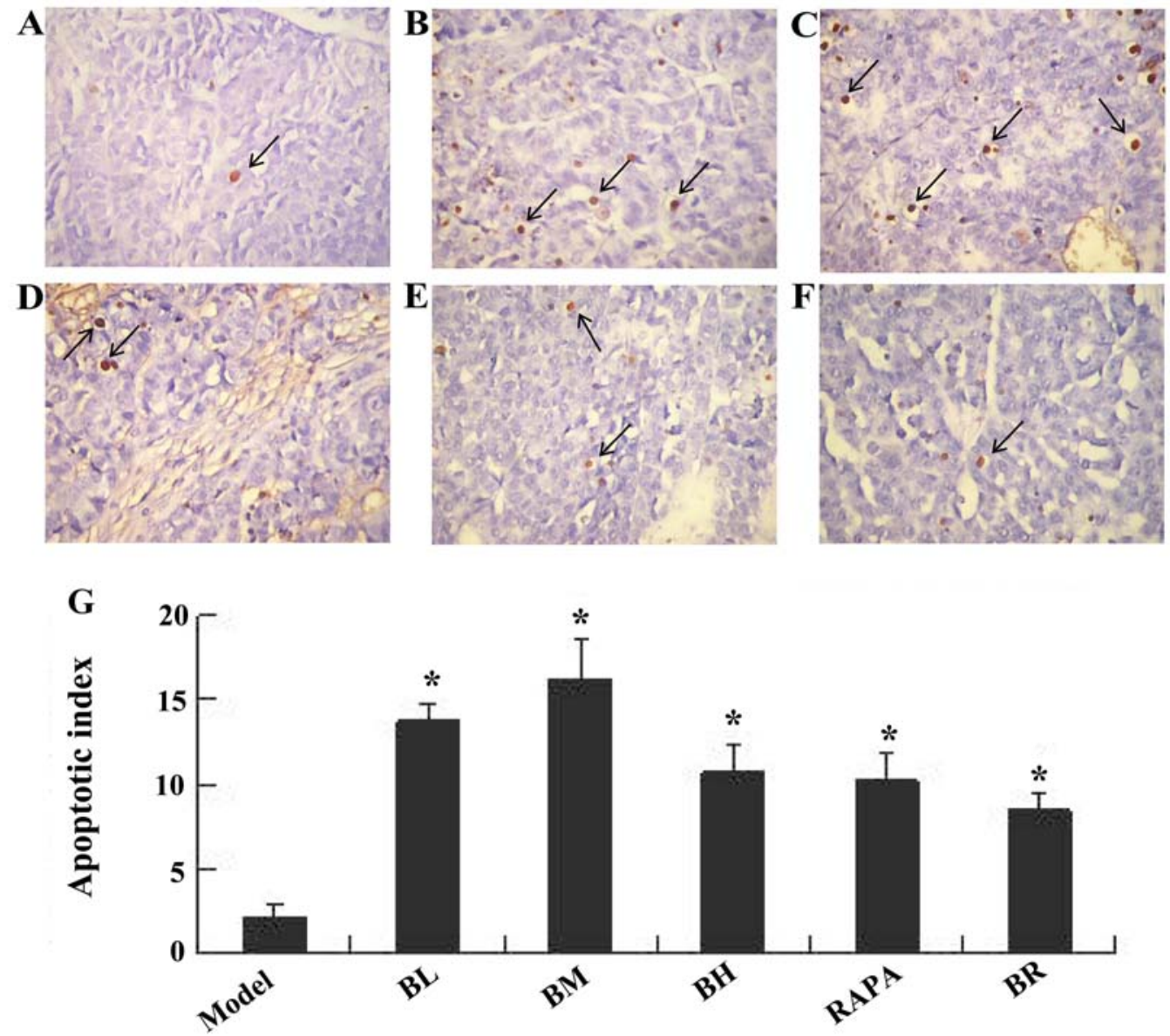

Figure 3. Apoptotic morphology of nude mice transplanted tumor cells, the arrow in the image refers to the apoptotic cells (by TUNEL labeling, $\mathrm{x} 40 \mathrm{magnifica-}$ tion). (A) Model; (B) Low-dose bufalin; (C) medium-dose bufalin; (D) high-dose bufalin; (E) rapamycin and (F) combination. (G) Apoptotic index of cells in transplanted tumors.

to exert anti-tumor, diuretic, cardiac, blood pressure-promoting, anesthetic, anti-inflammatory, anti-radiation and antitussive effects and has been shown to inhibit the $\mathrm{Na}^{+} / \mathrm{K}^{+}$-ATP enzyme (23). Krenn and Kopp suggested that the most effective composition of bufalin had digoxin-like immunoreactive effects (23). Bufalin is an effective antitumor agent and has been applied in the therapy of diverse malignant tumors (24). Studies have shown that cardiac glycoside drugs inhibit the proliferation of tumor cells and induce apoptosis in gastric cancer (25), prostate cancer (26), liver cancer (27), endometrial 

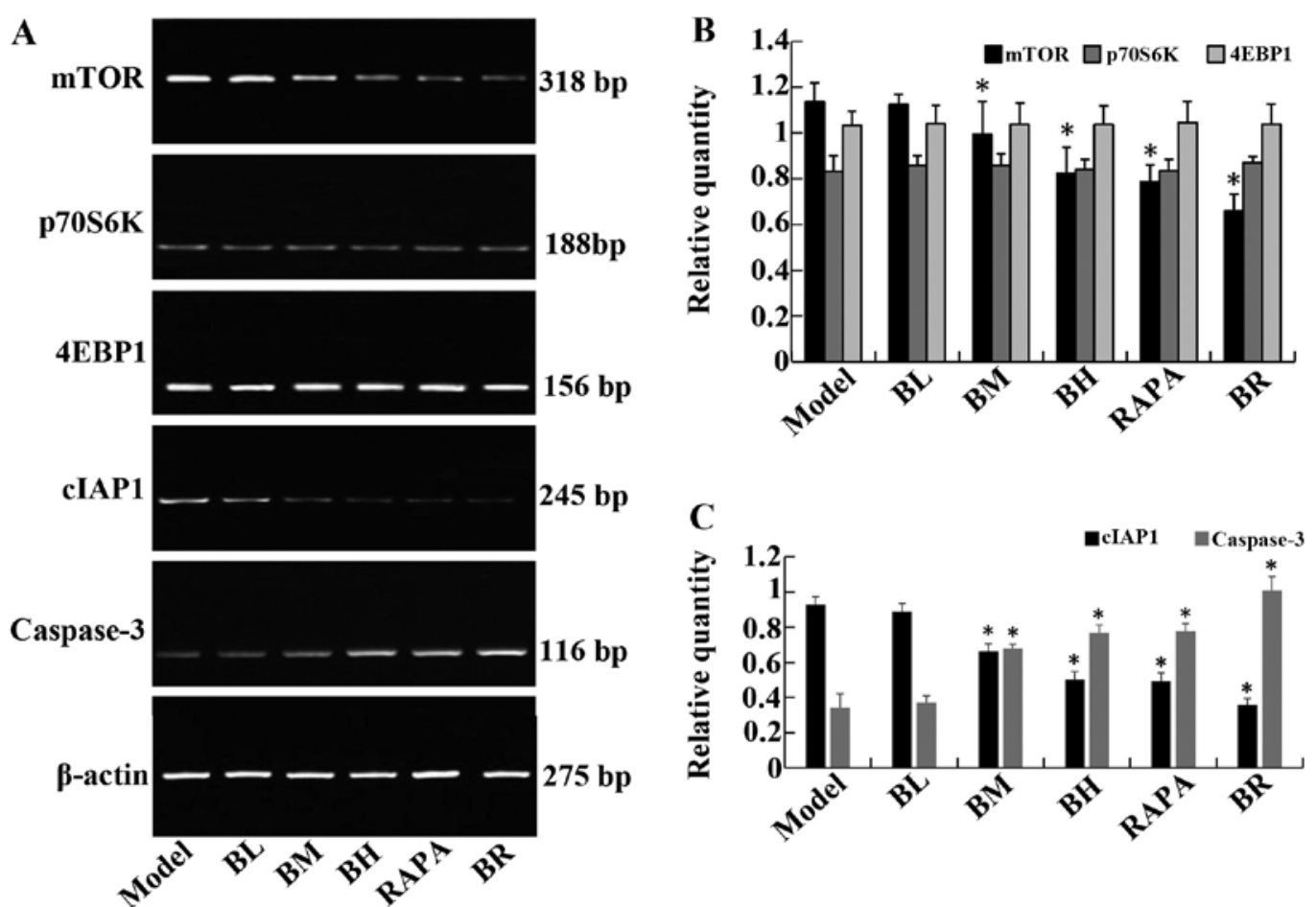

Figure 4. The expression of mTOR, P70S6K, 4EBP1, cIAP1 and caspase-3 mRNA in orthotopic transplanted tumor of human esophageal squamous cell carcinoma (ESCC) in nude mice. (A) RT-PCR analysis of mTOR, P70S6K, 4EBP1, cIAP1 and caspase-3 mRNA levels in each proup. (B) The relative expression of mTOR, P70S6K, 4EBP1 in each proup. (C) The relative expression of cIAP1 and caspase-3 in each proup. "P<0.05 vs. mode.
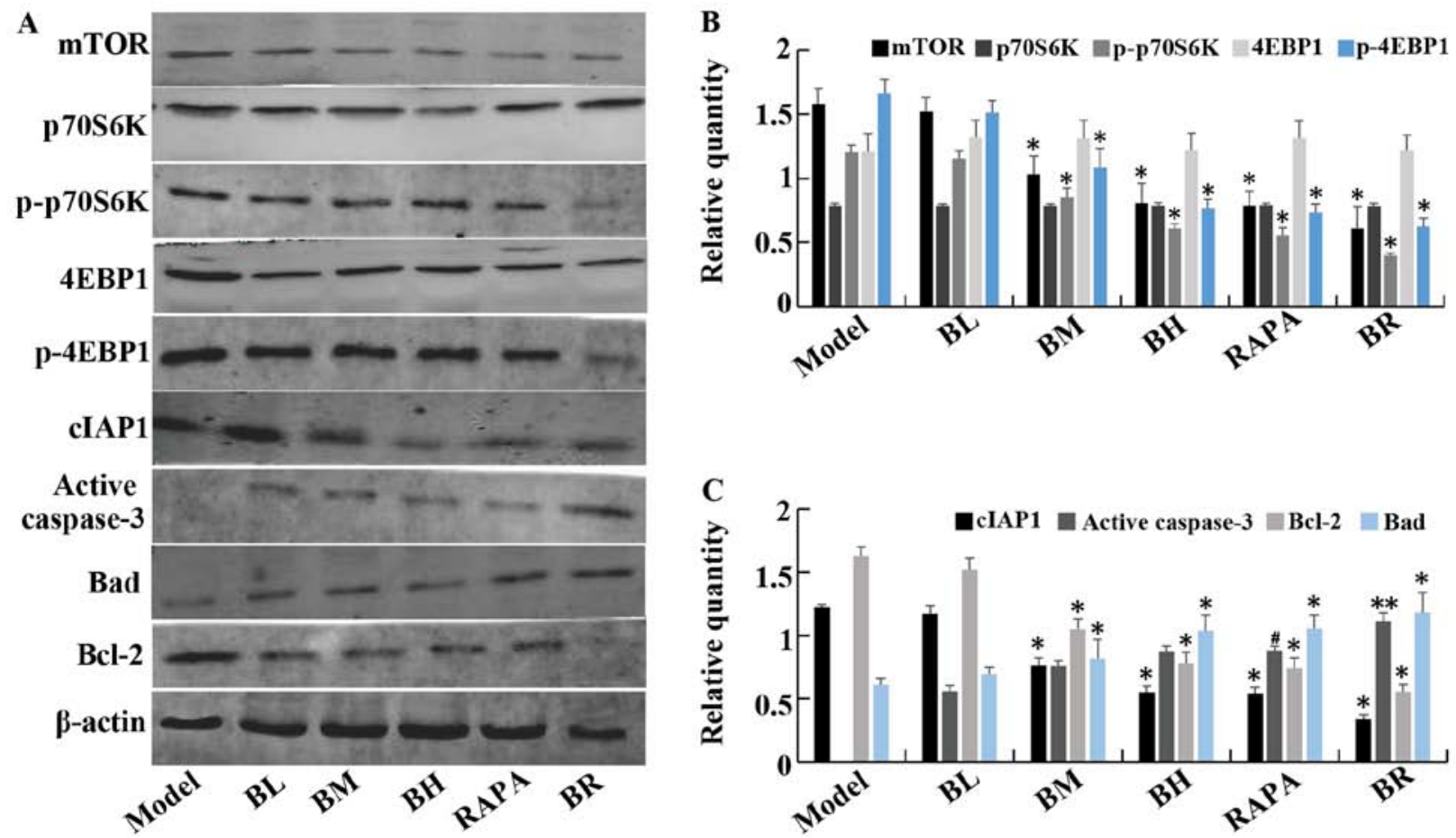

Figure 5. The expression of mTOR, P70S6K, p-P70S6K, 4EBP1, p-4EBP1, cIAP1, active caspase-3, Bad, Bcl-2 protein in orthotopic transplanted tumor of human esophageal squamous cell carcinoma (ESCC) in nude mice. (A) Western blotting of mTOR, P70S6K, p-P70S6K, 4EBP1, p-4EBP1, cIAP1, active caspase-3, Bad and Bcl-2 protein levels in each groups. (B) The relative expression of mTOR, P70S6K, p-P70S6K, 4EBP1, p-4EBP1 in each groups. (C) The relative expression of cIAP1, active caspase-3, Bad and Bcl-2 in each groups. ${ }^{* *} \mathrm{P}<0.05$ vs. model; ${ }^{~} \mathrm{P}<0.05$ vs. medium-dose bufalin; ${ }^{*} \mathrm{P}<0.05$ vs. rapamycin and high-dose bufalin.

cancer (28), ovarian cancer (28) and colon cancer (29). Lee et al found that bufalin inhibited tumor growth by inhibiting endothelial cell proliferation and angiogenesis (30). Xia et al found that the anti-tumor effects of bufalin were associated 

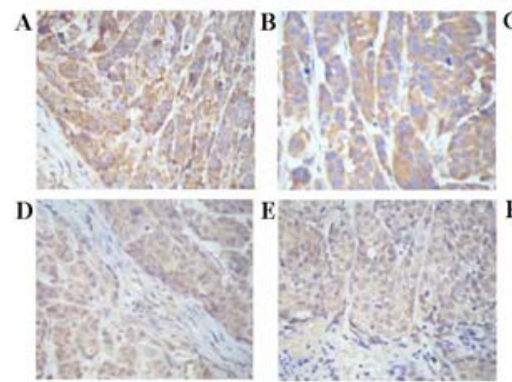

mTOR
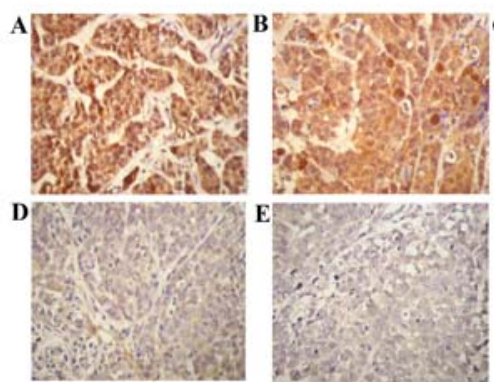

p-p70S6K
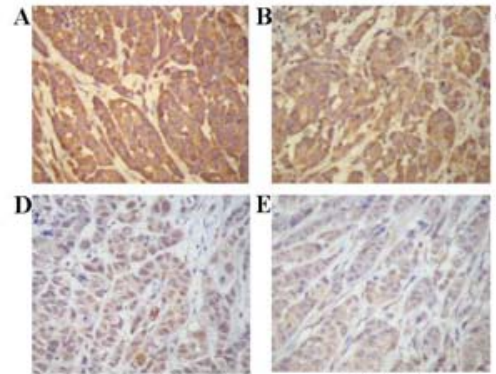

p-4EBP1
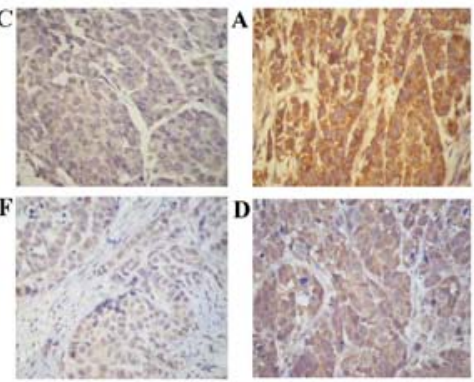

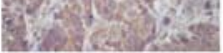

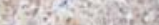

cIAP1
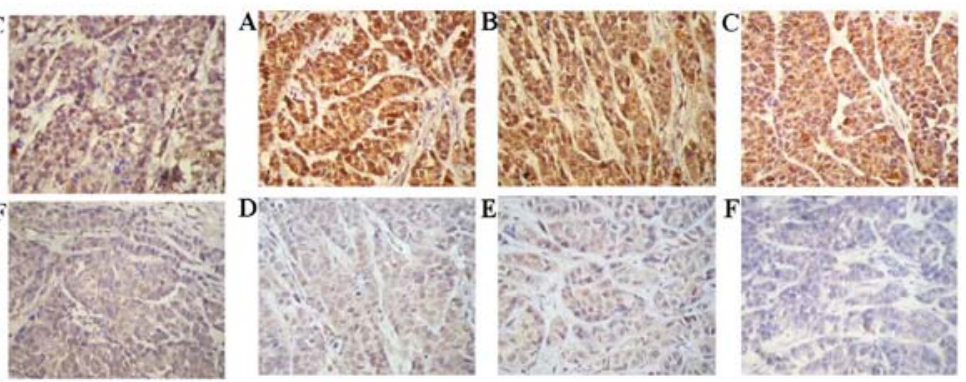

Bcl-2
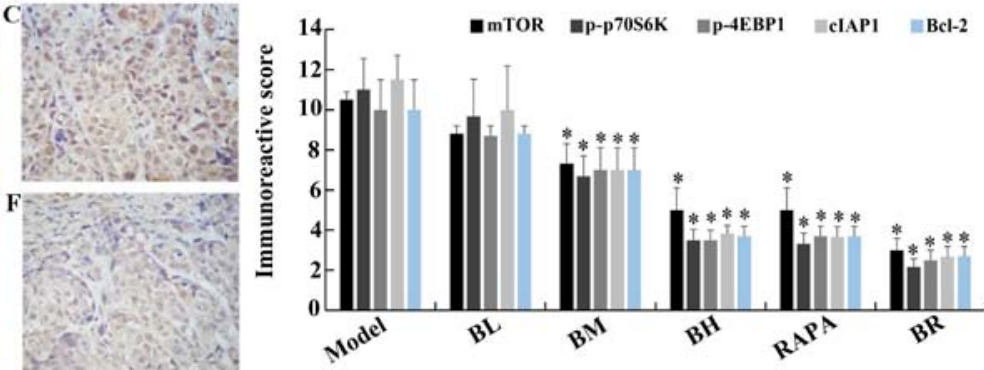

Figure 6. Immunohistochemical staining of mTOR, p-P70S6K, p-4EBP1, cIAP1 and Bcl-2 in nude mice transplanted tumor (x40 magnification). (A) Model; (B) low-dose bufalin; (C) medium-dose bufalin; (D) high-dose bufalin; (E) rapamycin and (F) combination.

with the induction of tumor cell apoptosis (31). Yamada et al found that the anti-tumor effects of bufalin were associated with the induction of tumor cell differentiation (32). Currently, the exact mechanisms responsble for the anti-tumor effects of bufalin are not clear. $\mathrm{Xu}$ et al indicated that bufalin is an active compound of the traditional Chinese medicine, Chansu, which exhibits significant anti-tumor activities in many solid tumors and leukemia cell lines (33). Qiu et al also demonstrated that bufalin inbibits the proliferation and invasion of hepatocellular carcinoma cells (34). Our study demonstrated that bufalin exerts anti-tumor effects. However, further research is required to fully determine the effects of bufalin in ESCC.

A previous study suggested that bufalin decreased the phosphorylation levels of extracellular signal-regulated kinase (ERK) in vitro, while the levels of non-phosphorylation ERK were not affected. It was suggested that the anti-tumor effects of bufalin were associated with the activation of ERK (35).

Numerous studies have confirmed that the mTOR/ p70S6K signaling pathway plays a central role in cell survival, growth and proliferation, and is significantly activated in a variety of tumors, such as breast cancer, prostate cancer and cervical cancer, and has become a novel target in cancer therapy (36-41). mTOR is a member of the PIKK superfamily and is an untypical serine/threonine protein kinase. It is highly conserved evolutionary (42). mTOR regulates protein synthesis, cell growth, and proliferation and apoptosis (43). p70S6K is an important key factor downstream of the mTOR pathway. It is a substrate which activates mTOR directly and promotes protein synthesis. p70S6K is phosphorylated into p-p70S6K through mTOR and the translational activity of p-p70S6K is approximately 100-fold greater than that of p70S6K (44). The activation process of p70S6K is very complex, and it requires at least 7 serine/threonine phosphorylation sites (45). These can be divided into two regions, one for Thr389, Thr229 and Ser404; phosphorylation at Thr389 could be directly activated protein kinase. The second is responsible for the regulation of kinase activity at the C-terminus (Ser411, Ser424, Ser418 and Thr421) (45).

$4 \mathrm{EBP} 1$ is a negative regulator of protein translation translation in mammalian cells. When cells are exposed to external stimuli, 4EBP1 is inactivated by activated mTOR which causes dissociation from eIF-4E (46). In the presence of mTOR inhibitors, 4EBP1 is dephosphorylated and combines with eIF-4E, inhibiting the initiation of translation. In our study, we measured the tumor volume in nude mice following treatment with low- or high-dose bufalin and bufalin in combination with 


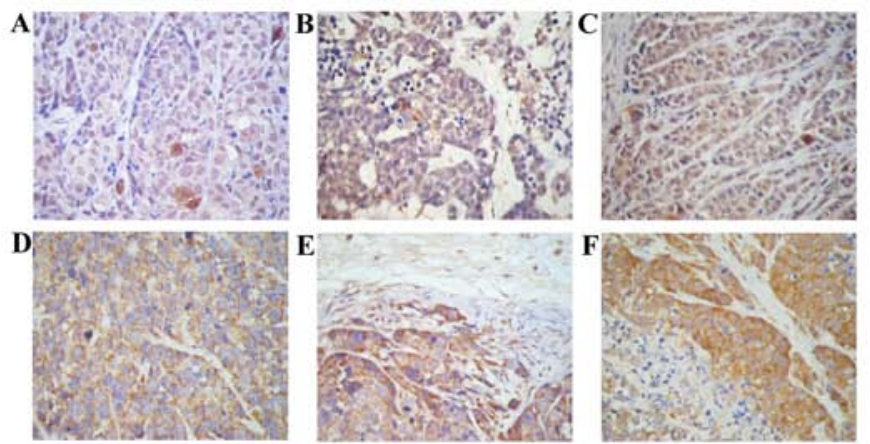

Active caspase-3

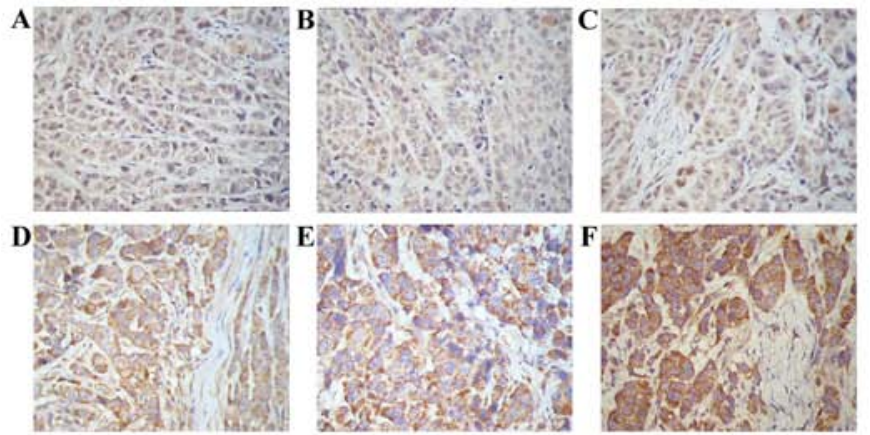

Bad

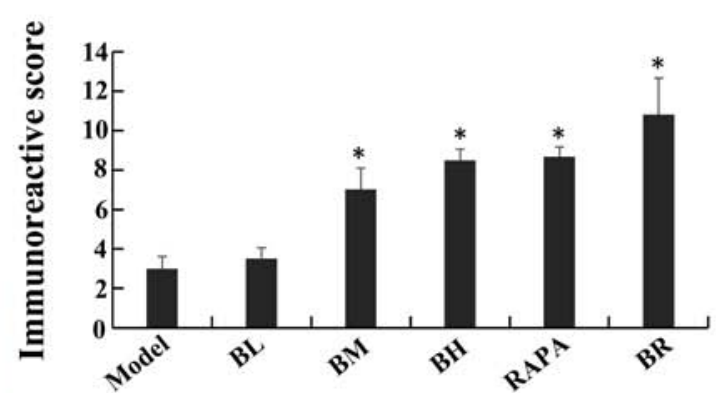

Active caspase-3

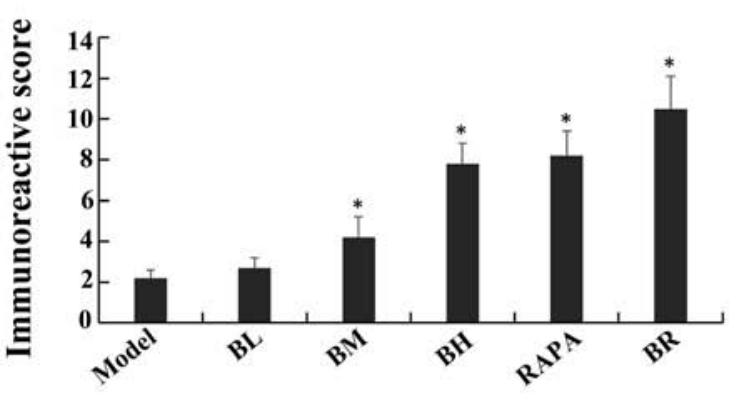

Bad

Figure 7. Immunohistochemical staining of active caspase-3, Bad in nude mice transplanted tumor (x40 magnification). (A) Model; (B) low-dose bufalin; (C) medium-dose bufalin; (D) high-dose bufalin; (E) rapamycin and (F) combination.

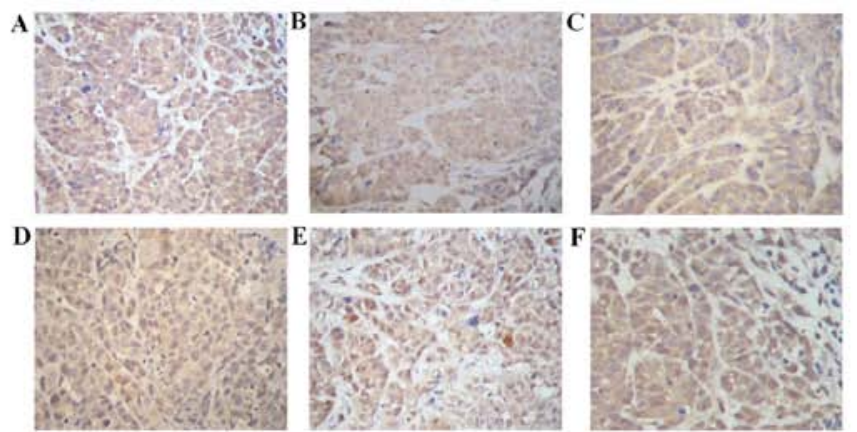

p70S6K

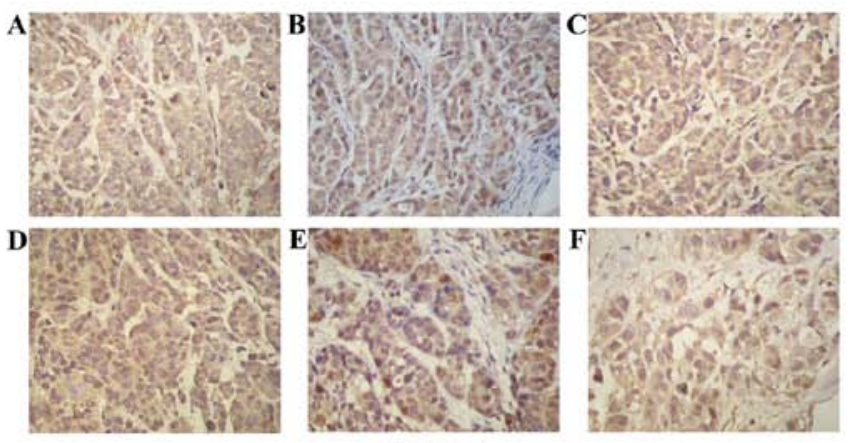

4EBP1

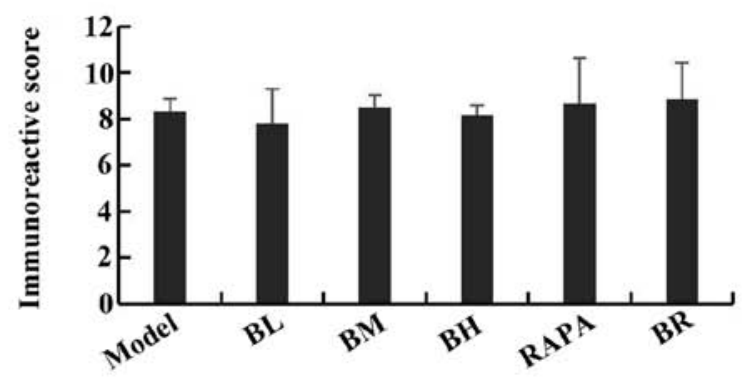

p70S6K

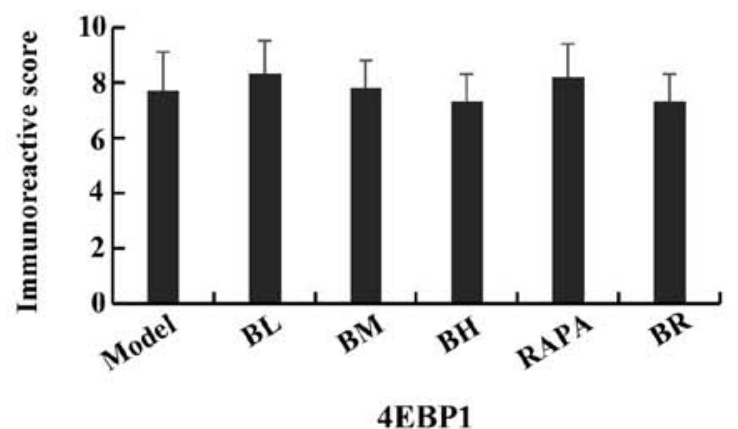

Figure 8. Immunohistochemical staining of P70S6K, 4EBP1 in nude mice transplanted tumor (x40 magnification). (A) Model; (B) low-dose bufalin; (C) medium-dose bufalin; (D) high-dose bufalin; (E) rapamycin and (F) combination.

rapamycin. Compared with the model group, tumor growth was decreased in the BM, BH, RAPA and BR groups, whereas tumor growth was not significantly altered in the BL group. This suggests that bufalin inhibits tumor growth at medium 
and high doses, whereas it has no significant inhibitory effect at lower doses. The results from western blot analysis and immunohistochemistry indicated that the expression of p-p70S6K and p-4EBP1 decreased following treatment with bufalin and/or rapamycin. The most significant decrease was observed in the BR (combination) group. Of note, the expression of p70S6K and 4EBP1 exhibited no significant change. We thus inferred that bufalin inhibited the phosphorylation and activity of p70S6K and 4EBP1 by inhibiting the mTOR/ p70S6K pathway. Bufalin and rapamycin may thus inhibit the mTOR/p70S6K pathway synergistically. Therefore, it can be concluded that the anti-tumor effects of bufalin are mediated via the $\mathrm{mTOR} / \mathrm{p} 70 \mathrm{~S} 6 \mathrm{~K}$ pathway and via the inhibition of the activity of p70S6K and 4EBP1. The results of RT-PCR demonstrated that the mRNA expression levels of p70S6K, 4EBP1 were not significantly altered in each group. Bufalin inhibited the phosphorylation of p70S6K, which further confirmed our above conclusions.

Recently, it has been shown that the occurrence of malignant tumor is associated with the dysregulation of apoptosis. Inhibitor of apoptosis proteins (IAPs) are a type of highly conserved proteins which regulate the activity of caspase-3/-7/-9 and immune signals to inhibit the process of apoptosis. cIAP1 inhibits apoptosis mainly by suppressing the activity of caspase-3/-7 and NF- $\mathrm{KB}$ (20). cIAP1 plays a pivotal role in a large number of malignancies. In China, the incidence of esophageal cancer is high and reports on cIAP1 in esophageal cancer are limited. Xu et al suggested that a high expression of cIAP1 in ESCC was associated with the chemosensitivity of esophageal cancer (47). Zhang et al found that the overexpression of XIAP in esophageal cancer and the downregulation of XIAP was associated with RNA interference and could enhance the chemotherapeutic sensitivity of esophageal cancer (48). In addition, caspase proteases exist in the cytoplasm and can cleave peptide bonds in aspartic acid residue of the target protein specifically (48). Caspase- 3 is the most critical terminal cleavage enzyme during apoptosis, which is the terminal executive molecule downstream of caspase cascade. Caspase- 3 is the intersection of the death receptor pathway and mitochondrial pathway. Thus, caspase- 3 is also known as the death protease (21). Normally, caspase-3 exists in the cell cytoplasm as zymogen form (pro-caspase) When the zymogen is stimulated by an apoptotic signal, pro-caspase can be activated to translate into active-caspase-3, which is involved in the process of apoptosis.

The Bcl-2 family plays a critical role in the process of apoptosis, as it consists of anti-apoptotic proteins (Bcl-2, Bcl-xL, Bcl-w and ced-9) and pro-apoptotic proteins (Bad, Bax, Bak and Bcl-xS). Bcl-2 is anti-apoptotic and can inhibit cell apoptosis, prolonging the life of cells, whereas Bad promotes cell apoptosis (49). In this study, apoptosis was detected by TUNEL assay. We found that the apoptotic index was highest in the BL and BM groups, it was decreased in the BH group. It was thus suggested that bufalin induces apoptosis mainly at low- and medium-doses, while it can kill tumor cells directly at high doses, leading to large areas of necrosis. In the RAPA and BR groups, large areas of necrosis were observed, but the apoptotic index was lower than that of the BL and BM groups. The results of RT-PCR, western blot analysis and immunohistochemistry revealed that the mRNA and protein expression of cIAP1 gradually decreased in each group, suggesting that bufalin exerted a dose-dependent effect. That is to say, with the increasing concentration of bufalin, the expression of cIAP1 decreased, being lowest in the BR group. Simultaneously, the expression of caspase-3 mRNA and active caspase-3 protein increased in each group. With the inreasing concentrations of bufalin, the expression of Bad increased, whereas that of Bcl-2 decreased. Thus, bufalin exerted pro-apoptotic effects by regulating the expression levels of $\mathrm{Bcl}-2$ and Bad. There was a certain dose-dependent effect. We speculated that bufalin and rapamycin induced apoptosis synergistically. The anti-tumor effects of bufalin may be associated with the down-regulation of cIAP1 and the upregulation of of caspase-3 in esophageal cancer cells. As cIAP1 can inhibit the activation of caspase-3, with the decrease in the expression of cIAP1, the caspase- 3 was activated in the esophageal cancer cells. In this way, caspase- 3 is activated and can promote the initiation of the apoptotic process in esophageal cancer cells.

In conclusion, our study demonstrates tht bufalin exerts palpable anti-tumor effects on orthotopically transplanted ESCC tumors in nude mice. Bufalin decreased the levels of phosphorylated p70S6K and 4EBP1, whereas the levels of p70S6K and 4EBP1 were not affected. We thus suggest that bufalin inhibits tumor growth by suppressing the activation of p70S6K and 4EBP1. Bufalin also decreased the expression of cIAP1 and Bcl-2, and it upregulated active caspase- 3 and Bad simultaneously. Thus, the induction of tumor cell apoptosis may be one of the critical antitumor mechanisms of bufalin.

\section{Acknowledgements}

This study was supported by the National Natural Science Foundation of China. The authors would like to thank the research specialist staff of Laboratory Animal Center at the Fourth Hospital of Hebei Medical University. Finally, we would also like to thank the professors and technical staff of the Department of Pathology in the Fourth Hospital of Hebei Medical University.

\section{References}

1. Ferlay J, Shin HR, Bray F, Forman D, Mathers C and Parkin DM: Estimates of worldwide burden of cancer in 2008: GLOBOCAN 2008. Int J Cancer 127: 2893-2917, 2010.

2. Rustgi AK and El-Serag HB: Esophageal carcinoma. N Engl J Med 371: 2499-2509, 2014.

3. Siewert JR and Ott K: Are squamous and adenocarcinomas of the esophagus the same disease? Semin Radiat Oncol 17: 38-44, 2007.

4. Ou Y, Ma L, Ma L, Huang Z, Zhou W, Zhao C, Zhang B, Song Y, $\mathrm{Yu} \mathrm{C}$ and Zhan Q: Overexpression of cyclin B1 antagonizes chemotherapeutic-induced apoptosis through PTEN/Akt pathway in human esophageal squamous cell carcinoma cells. Cancer Biol Ther 14: 45-55, 2013.

5. Tong Q, Zhang W, Jin S, Li S and Chen Z: The relationship between $\mathrm{p} 27$ (kip1) expression and the change of radiosensitivity of esophageal carcinoma cells. Scand J Gastroenterol 46: 173-176, 2011.

6. Imoto I, Yang ZQ, Pimkhaokham A, Tsuda H, Shimada Y, Imamura M, Ohki M and Inazawa J: Identification of cIAP1 as a candidate target gene within an amplicon at 11q22 in esophageal squamous cell carcinomas. Cancer Res 61: 6629-6634, 2001.

7. Dhar R, Persaud SD, Mireles JR and Basu A: Proteolytic cleavage of $\mathrm{p} 70$ ribosomal S6 kinase by caspase-3 during DNA damage-induced apoptosis. Biochemistry 48: 1474-1480, 2009.

8. Fingar DC, Richardson CJ, Tee AR, Cheatham L, Tsou C and Blenis J: mTOR controls cell cycle progression through its cell growth effectors S6K1 and 4E-BP1/eukaryotic translation initiation factor 4E. Mol Cell Biol 24: 200-216, 2004. 
9. Neufeld TP: TOR-dependent control of autophagy: biting the hand that feeds. Curr Opin Cell Biol 22: 157-168, 2010.

10. Ganley IG, Lam du H, Wang J, Ding X, Chen S and Jiang X: ULK1.ATG13.FIP200 complex mediates mTOR signaling and is essential for autophagy. J Biol Chem 284: 12297-1305, 2009.

11. Zinzalla V, Stracka D, Oppliger W and Hall MN: Activation of mTORC2 by association with the ribosome. Cell 144: 757-768, 2011.

12. Oh WJ, Wu CC, Kim SJ, Facchinetti V, Julien LA, Finlan M, Roux $\mathrm{PP}, \mathrm{Su} \mathrm{B}$ and Jacinto E: mTORC2 can associate with ribosomes to promote cotranslational phosphorylation and stability of nascent Akt polypeptide. EMBO J 29: 3939-3951, 2010.

13. Cornu M and Hall MN: mTORC1 and mTORC2 in energy homeostasis. In: The Enzymes: Structure, Function and Regulation of TOR Complexes from Yeasts to Mammals. Tamanoi $\mathrm{F}$ and Hall MN (eds). Academic Press, Amsterdam, pp263-278, 2010.

14. Hanahan D and Weinberg RA: The hallmarks of cancer. Cell 100: 57-70, 2000.

15. Hanahan D and Weinberg RA: Hallmarks of cancer: the next generation. Cell 144: 646-674, 2011

16. Um SH, Frigerio F, Watanabe M, Picard F, Joaquin M, Sticker M, Fumagalli S, Allegrini PR, Kozma SC, Auwerx J and Thomas G: Absence of S6K1 protects against age- and diet-induced obesity while enhancing insulin sensitivity. Nature 431: 200-205, 2004.

17. Ly C, Arechiga AF, Melo JV, Walsh CM and Ong ST: Bcr-Abl kinase modulates the translation regulators ribosomal protein S6 and 4E-BP1 in chronic myelogenous leukemia cells via the mammalian target of rapamycin. Cancer Res 63: 5716-5722, 2003

18. Kharas MG, Deane JA, Wong S, O'Bosky KR, Rosenberg N, Witte ON and Fruman DA: Phosphoinositide 3-kinase signaling is essential for $\mathrm{ABL}$ oncogene-mediated transformation of B-lineage cells. Blood 103: 4268-4275, 2004.

19. Liu Y and Li X: The research progress of p70S6K1 and 4E-BPs of the AKt/mTOR signal pathway in promoting the occurrence of tumor. J Modern Oncol 24: 847-850, 2016.

20. Xu Y, Liu F, Zhou LP and Zhao XH: c-IAP1 expression and tumor chemosensitivity in esophageal squamous cell carcinoma. World Chin J Digestol 19: 1138-1144, 2011 (In Chinese).

21. Wang Q, Huang Y, Ni Y, Wang H and Hou Y: siRNA targeting midkine inhibits gastric cancer cells growth and induces apoptosis involved caspase-3,8,9 activation and mitochondrial depolarization. J Biomed Sci 14: 783-795, 2007.

22. Liu MY, Hou GQ, Zhang Y, Bei WJ and Yan AH: Effects of mTOR siRNA on mTOR/p70S6K signaling pathway in esophageal squamous cell carcinoma cells and the growth of transplanted tumor in nude mice. Zhonghua Zhong Liu Za Zhi 33: 334-339, 2011 (In Chinese).

23. Krenn L and Kopp B: Bufadienolides from animal and plant sources. Phytochemistry 48: 1-29, 1998.

24. Yin PH, Liu X, Qiu YY, Cai JF, Qin JM, Zhu HR and Li Q: Anti-tumor activity and apoptosis-regulation mechanisms of bufalin in various cancers: new hope for cancer patients. Asian Pac J Cancer Prev 13: 5339-5343, 2012.

25. Li D, Qu X, Hou K, Zhang Y, Dong Q, Teng Y, Zhang J and Liu Y: PI3K/Akt is involved in bufalin-induced apoptosis in gastric cancer cells. Anticancer Drugs 20: 59-64, 2009.

26. Zhai XF, Fang FF, Liu Q, Meng YB, Guo YY and Chen Z: miR-181a contributes to bufalin-induced apoptosis in $\mathrm{PC}-3$ prostate cancer cells. BMC Complement Altern Med 13: 325, 2013.

27. Qi F, Inagaki Y, Gao B, Cui X, Xu H, Kokudo N, Li A and Tang W: Bufalin and cinobufagin induce apoptosis of human hepatocellular carcinoma cells via Fas- and mitochondria-mediated pathways. Cancer Sci 102: 951-958, 2011.

28. Takai N, Ueda T, Nishida M, Nasu K and Narahara H: Bufalin induces growth inhibition, cell cycle arrest and apoptosis in human endometrial and ovarian cancer cells. Int J Mol Med 21: 637-643, 2008

29. Xie CM, Chan WY, Yu S, Zhao J and Cheng CH: Bufalin induces autophagy-mediated cell death in human colon cancer cells through reactive oxygen species generation and JNK activation. Free Radic Biol Med 51: 1365-1375, 2011.

30. Lee DY, Yasuda M, Yamamoto T, Yoshida T and Kuroiwa Y: Bufalin inhibits endothelial cell proliferation and angiogenesis in vitro. Life Sci 60: 127-134, 1997.
31. Xia J, Jiang $\mathrm{M}$ and Jiang Y: The research progress of the anti-tumor effect of toad venom and its effective components in vitro. Modern Oncol 16: 1441-1444, 2008 (In Chinese).

32. Yamada K, Hino K, Tomoyasu S, Honma Y and Tsuruoka N: Enhancement by bufalin of retinoic acid-induced differentiation of acute promyelocytic leukemia cells in primary culture. Leuk Res 22: 589-595, 1998

33. Xu Y, Chen M, Jin XF, Qian C, Xu XM and Zhang X: Research progress of in vitro and in vivo anti-tumor effects and formulation of bufalin. Zhongguo Zhong Yao Za Zhi 39: 2829-2833, 2014 (In Chinese)

34. Qiu DZ, Zhang ZJ, Wu WZ and Yang YK: Bufalin, a component in Chansu, inhibits proliferation and invasion of hepatocellular carcinoma cells. BMC Complement Altern Med 13: 185, 2013.

35. Ding Y, Wang XL and Deng HY: Mechanism of bufalin affecting the proliferation and migration of human esophageal carcinoma cells through inhibiting the activity of Raf/MEK/ERK pathway. Chinese General Practice 21, 2015 (In Chinese).

36. Easley CA IV, Ben-Yehudah A, Redinger CJ, Oliver SL, Varum ST, Eisinger VM, Carlisle DL, Donovan PJ and Schatten GP: mTOR-mediated activation of p70 S6K induces differentiation of pluripotent human embryonic stem cells. Cell Reprogram 12: 263-273, 2010.

37. Smolewski P: Recent developments in targeting the mammalian target of rapamycin (mTOR) kinase pathway. Anticancer Drugs 17: 487-494, 2006.

38. Zhang YJ, Dai Q, Sun DF, Xiong H, Tian XQ, Gao FH, Xu MH, Chen GQ, Han ZG and Fang JY: mTOR signaling pathway is a target for the treatment of colorectal cancer. Ann Surg Oncol 16: 2617-2628, 2009 (In Chinese).

39. Li G, Shan C, Liu L, Zhou T, Zhou J, Hu X, Chen Y, Cui H and Gao N: Tanshinone IIA inhibits HIF-1 $\alpha$ and VEGF expression in breast cancer cells via mTOR/p70S6K/RPS6/4E-BP1 signaling pathway. PLoS One 10: e0117440, 2015 (In Chinese).

40. Saraswati S, Kumar S and Alhaider AA: $\alpha$-santalol inhibits the angiogenesis and growth of human prostate tumor growth by targeting vascular endothelial growth factor receptor 2-mediated AKT/mTOR/P70S6K signaling pathway. Mol Cancer 12: 147, 2013.

41. Shin JM, Jeong YJ, Cho HJ, Park KK, Chung IK, Lee IK, Kwak JY, Chang HW, Kim CH, Moon SK, et al: Melittin suppresses HIF-1 $\alpha /$ VEGF expression through inhibition of ERK and $\mathrm{mTOR} / \mathrm{p} 70 \mathrm{~S} 6 \mathrm{~K}$ pathway in human cervical carcinoma cells. PLoS One 8: e69380, 2013 (In Chinese).

42. Zheng J: mTOR signal pathway and tumor. Life Sciences 18: 261-265, 2006.

43. Pan Z, Zhang L and Jiang J: The research progress of mTOR. Chin J Cell Biol 28: 395-398, 2006.

44. Jefferies HB, Fumagalli S, Dennis PB, Reinhard C, Pearson RB and Thomas G: Rapamycin suppresses 5'TOP mRNA translation through inhibition of p70 s6k. EMBO J 16: 3693-3704, 1997.

45. Cheng HD, Zhao QN, Song WD, Sarengaowa and Liu F: The current research of the component p-P70S6K of mTOR signal pathway and colon cancer. Journal of Disease Monitor and Control 6: 78-79, 2012.

46. Asimomytis A, Karanikou M, Rodolakis A, Vaiopoulou A, Tsetsa P, Creatsas G, Stefos T, Antsaklis A, Patsouris E and Rassidakis GZ: mTOR downstream effectors, 4EBP1 and eIF4E, are overexpressed and associated with HPV status in precancerous lesions and carcinomas of the uterine cervix. Oncol Lett 12: 3234-3240, 2016

47. Xu Y and Zhao XH: IAP family and IAP-targeted therapy. Life Sciences 22: 161-168, 2010.

48. Zhang S, Ding F, Luo A, Chen A, Yu Z, Ren S, Liu Z and Zhang L: XIAP is highly expressed in esophageal cancer and its downregulation by RNAi sensitizes esophageal carcinoma cell lines to chemotherapeutics. Cancer Biol Ther 6: 973-980, 2007.

49. Cong YM, Liu P, Jin DP, et al: The relationship of Bcl-2 family with cell apoptosis. J Chin Med Res 8: 125-127, 2008 (In Chinese). 